\title{
Multifunctional Indocyanine Green Applications for Fluorescence-Guided Laparoscopic Colorectal Surgery
}

\author{
Gyung Mo Son ${ }^{1,2,3}$, Hong-min $\mathrm{Ahn}^{1}$, In Young Lee ${ }^{2,3}$, Gi Won $\mathrm{Ha}^{4}$ \\ ${ }^{1}$ Department of Surgery, Pusan National University Yangsan Hospital, Yangsan; ${ }^{2}$ Research Institute for Convergence of Biomedical Science and \\ Technology, Pusan National University Yangsan Hospital, Yangsan; ${ }^{3}$ Medical Research Center, Pusan National University School of Medicine, \\ Yangsan; ${ }^{4}$ Research Institute of Clinical Medicine of Jeonbuk National University-Biomedical Research Institute of Jeonbuk National \\ University Hospital, Jeonju, Korea
}

Indocyanine green (ICG) could be applied for multiple functions such as fluorescent tumor localization, fluorescence lymph node mapping (FLNM), and intraoperative angiography in colorectal cancer surgery. With the near-infrared (NIR) systems, colonoscopic ICG tattooing can be used to define the early colorectal cancer that cannot be easily distinguished through the serosal surface. The lymphatic pathways can be visualized under the NIR system when ICG is injected through the submucosal or subserosal layer around the tumor. Intraoperative ICG angiography can be applied to find a favorable perfusion segment before the colon transection. Although all fluorescence functions are considered essential steps in image-guided surgery, it is difficult to perform multifunctional ICG applications in a single surgical procedure at once because complex protocols could interfere with each other. Therefore, we review the multifunctional ICG applications for fluorescent tumor localization, FLNM, and ICG angiography. We also discuss the optimal protocol for fluorescence-guided colorectal surgery.

Keywords: Tattooing; Fluorescent dyes; Fluorescein angiography; Laparoscopy; Colorectal neoplasms

\section{INTRODUCTION}

Colorectal cancer is one of the most common gastrointestinal cancer. Laparoscopic and robotic surgeries are performed increasingly with the advance of surgical technique and the development of surgical device technology. However, the localization of earlystage colorectal cancer is still challenging in the field of minimally invasive surgery without tactile sensation [1]. To localize the small tumor, many intraoperative navigation methods have been introduced, including intraoperative ultrasound and preoperative endoscopic tattooing [2].

Received: Feb 22, 2021 - Revised: Apr 29, 2021 - Accepted: May 7, 2021 Correspondence to: Gyung Mo Son, M.D.

Department of Surgery, Pusan National University Yangsan Hospital, 20 Geumo-ro, Mulgeum-eup, Yangsan 50612, Korea

Tel: +82-55-360-2124, Fax: +82-55-360-2154

E-mail:skm1711@pusan.ac.kr

ORCID: https://orcid.org/0000-0002-8861-6293

(C) 2021 The Korean Society of Coloproctology

This is an open-access article distributed under the terms of the Creative Commons Attribution NonCommercial License (https://creativecommons.org/licenses/by-nc/4.0) which permits unrestricted noncommercial use, distribution, and reproduction in any medium, provided the original work is properly cited.
Indocyanine green (ICG) is a tricarbocyanine iodide dye molecule that is amphiphilic, relatively nontoxic. ICG is the only safe fluorescence agent approved by the U.S. Food and Drug Administration (FDA) for usage in clinical applications. ICG can be used either intravenous or tissue-directed injections. Intravenously injected ICG rapidly binds to plasma proteins, such as albumins, globulins, and lipoproteins, thus approximately $95 \%$ to $98 \%$ of ICG remains within the bloodstream [3].

In colorectal cancer surgery, ICG could be applied for multiple functions such as fluorescent tumor localization, fluorescence lymph node mapping (FLNM), and intraoperative angiography for image-guided surgery [3-5]. With the near-infrared (NIR) imaging systems, the ICG can be used to localize the early colorectal cancer site that cannot be easily distinguished through the serosal surface, especially after endoscopic mucosal resection or submucosal dissection [6]. The lymphatic pathways can be also visualized under the NIR image system when ICG is injected through the submucosal or subserosal layer around the tumor $[7,8]$. Intraoperative ICG angiography could be applied to find a favorable perfusion segment before the colon transection. By facilitating the perfusion at the anastomosis, ischemia-related anastomotic com- 
plications could be prevented [9].

Even though all of ICG fluorescence functions, including tumor localization, lymph node mapping, and intraoperative angiography, can be considered as essential steps in the image-guided surgery, it is difficult to perform multifunctional ICG applications in a single surgical procedure at once because complex and unstandardized ICG protocols can interfere with each other. Conventional ICG tattooing protocol is useful for tumor localization through the naked eye exploration, but unsuitable for fluorescence-guided surgery because conventional ICG dosage is usually more than $25 \mathrm{mg}$ that emits fluorescence in the entire intraperitoneal cavity under the NIR camera image. In this situation, ICG could not be used for intraoperative angiography or FLNM. Preoperative ICG tattooing protocols vary between facilities and surgeons and there is still a lack of research on the standardized ICG tattooing protocol for multifunctional applications in fluorescence-guided laparoscopic colorectal surgery.

This study aimed to review the multifunctional ICG applications for fluorescent tumor localization, ICG angiography, and FLNM within a single surgery and discuss the optimal protocol for fluorescence-guided laparoscopic colorectal surgery.

\section{PROTOCOLS OF ICG APPLICATIONS}

\section{Fluorescent tumor localization}

In our facility, patients are admitted to our facility 2 days before surgery for preoperative colonoscopic ICG tattooing. The endoscopic submucosal tattooing is performed 16 to 18 hours before surgery. Diluted ICG solution $(0.25 \mathrm{mg} / \mathrm{mL})$ is directly injected into the submucosal layer of the colon or rectum that was slightly distal to the tumor. The submucosal ICG injection $(0.25 \mathrm{mg} / \mathrm{mL}$, $1 \mathrm{~mL}$ ) is administered at 2 or more different sites close to the distal side of cancer by an endoscopist. After the trocar insertion, the tumor localization is evaluated in the white light view of the laparoscopic camera, and the gross localization of blue-colored tattooing lesion is explored at first. Using the same aspect, the NIR camera is changed to ICG image mode to identify the fluorescent tumor localization. There are 3 different kinds of laparoscopic fluorescence imaging systems: Stryker (1588 AIM camera system, Stryker, Kalamazoo, MI, USA), Storz (IMAGE1 S, Karl Storz, Tuttlingen, Germany), and Olympus (CLV-S200-IR, Olympus, Tokyo, Japan). These NIR camera systems use different fluorescence emission sources. Stryker uses a laser as the light source, whereas the other 2 imaging systems use xenon lamps. The selection of NIR camera systems depended on the available instrument conditions on the day of surgery.

Fluorescence lymph node mapping using indocyanine green FLNM is explored before initial lymph node dissection using the ICG image mode. The definite circular or crescent-shaped fluorescent lymph nodes could be found and that is considered as successful FLNM. Sometimes, we could meet no fluorescent lymph nodes as failed FLNM. During the radical D3 lymphadenectomy, the ICG stained lymph node might be placed on the extraterritorial area of the conventional D3 lymph node region, such as paraaortic nodes at that time, a surgeon must decide to extend lymph node dissection to remove extraterritorial ICG lymph nodes, such as en bloc resection or "berry picked" excision. After radical lymph node dissection is completed, the absence of any residual fluorescent lymph node is confirmed by using ICG mode of laparoscopic NIR camera.

In our facility, the submucosal ICG injection has been performed a day before surgery with preoperative colonoscopy not only for tattooing the tumor but also for the FLNM. Comparing with other protocols such as intraoperative subserosal injection or direct injection into dentate line before the rectal cancer surgery, this method seems inconvenient for the patients, since extra procedures have to be performed before the surgery. Nevertheless, the submucosal injection of ICG via preoperative colonoscopy has greater advantages on accurate ICG instillation into the submucosal layer and performing multifunctional applications of ICG. In our recent study, reduced ICG tattooing dosage let cancer be visualized clearly by the NIR system [10]. In addition, performing the FLNM and intraoperative ICG angiography may not be disturbed with surrounding ICG stained tissues.

\section{Intraoperative ICG angiography}

Intraoperative ICG angiography is performed to define a favorable perfusion segment before colon transection. The diluted ICG is prepared by the anesthesiologist as the dilution of ICG $(25 \mathrm{mg})$ in $10 \mathrm{~mL}$ of distilled water. The patient's body weight-adjusted ICG dosage $(0.05 \mathrm{mg} / \mathrm{kg})$ is injected slowly for 10 seconds. Colonic fluorescence intensity is monitored for 2 minutes after the intravenous ICG injection using the ICG mode of the laparoscopic NIR camera [7]. The ICG angiography could be performed either on the inside or outside the abdomen with "dark" surrounding conditions without room lighting. The optimal distance between the NIR camera lens and colon is considered as 4 to $5 \mathrm{~cm}$ for quantitative perfusion analysis [11]. The distance between the NIR camera lens and colon can be easily measured using the surgical paper ruler. Measurement of the reflection distance of laser is technically possible but is not yet commercially compatible with the surgical device. Usually, when the short axis of the colon is half of the monitor screen, the distance with the camera is maintained at 4 to $5 \mathrm{~cm}$ for optimal fluorescence intensity.

\section{MULTIFUNCTIONAL ICG APPLICATIONS}

\section{Optimal indocyanine green tattooing dosage for tumor localization and fluorescence lymph node mapping}

ICG is grossly a dark blue pigmented substance that is used for tumor tattooing. However, there is a lot of difficulty in observing blue-colored dye with the naked eye because ICG is easily washed out due to its small-sized molecular structure. Some previous 
studies reported ICG as less useful among other tattooing agents such as India ink with causing mucosal ulceration and inflammation $[12,13]$. With the fluorescence property of ICG and the development of the surgical NIR camera system, the tumor location and lymph nodes can easily be visualized under ICG image mode even with the diluted dosage that makes the tumor be hardly seen under white lights [13].

All clinical functions of ICG such as tumor localization, FLNM, and ICG angiography could play important roles in laparoscopic colorectal surgery [14]. Nevertheless, ICG tattooing with the conventional ICG dosage (25-50 mg) makes it impossible to distinguish the colon from surrounding tissues under the NIR camera, which may lead to failed ICG angiography and fluorescence localization. To avoid interference with stained surrounding tissues, the ICG tattooing dosage should be suitably diluted for successful fluorescent tumor localization, FLNM, and ICG angiography. In our clinical experiences with almost 200 patients, we had reduced ICG tattooing dosage by diluting the concentrate, and measured success or failure on each application; fluorescent tumor localization, FLNM, and intraoperative ICG angiography. From the results, we could have established the optimal ICG tattooing protocol; preoperative endoscopic submucosal injection near the tumor at ICG dosage of $0.5 \mathrm{mg}$, which improve the success rate of FLNM without the interference of fluorescent tumor localization and ICG angiography [10].

\section{Optimal indocyanine green angiography protocol}

The intraoperative ICG angiography technique could show realtime colon perfusion status during laparoscopic or robotic surgery and that could help colorectal surgeons to detect ischemic change at the colonic anastomosis site before colon transection. There are many studies on the clinical effect of ICG angiography to predict the risk of hypoperfusion at the anastomosis and preventing effect of anastomotic leakage [3, 9, 15-22]. The colonic ICG angiography protocols were summarized in Table 1 [9, 15-22]. All studies used intravenous ICG and NIR systems. However, surrounding conditional factors such as distance and surrounding lighting were mostly omitted. Many conditional factors affect the fluorescence intensity during ICG angiography, so we need to establish the optimal protocol for standardization of ICG angiography.

Fluorescence emitting sources are diverse; xenon lamp, laser, and light-emitting diode. The lighting conditions of the surgical field or the location of video shooting, either the intraabdominal or the extraabdominal space, can also affect the fluorescence intensity. The distance between the target colon and the laparoscopic camera lens is considered to be the most important factor causing differences in fluorescence intensity. Thus, several conditions like the fluorescence light source, color processing mode, illumination of the operating room, and the distance from the camera are all critical factors affecting the fluorescence intensity [9].

In our in vitro study, the distance from the camera to the subject affects the fluorescence intensity inversely. In the quantitative analysis, the fluorescence intensity tends to decrease when the distance increased between the laparoscopic camera and ICG test tube. Interestingly, the fluorescence intensity graph pattern has an inflection point around 4 to $5 \mathrm{~cm}$ of distance. This transitional section could be considered as an optimal distance zone for ICG angiography. So, the best shooting distance is considered as 4 to 5 $\mathrm{cm}$. Therefore, we are suggesting that 4 to $5 \mathrm{~cm}$ can be the optimal distance of ICG angiography for quantitative analysis [11].

Table 1. The protocols of intraoperative ICG angiography

\begin{tabular}{|c|c|c|c|c|c|c|c|c|}
\hline Reference & Year & $\begin{array}{l}\text { No. of } \\
\text { patients }\end{array}$ & $\begin{array}{l}\text { Types of } \\
\text { surgery }\end{array}$ & $\begin{array}{l}\text { Intravenous } \\
\text { dose of ICG }\end{array}$ & $\begin{array}{l}\text { Distance } \\
\text { (cm) }\end{array}$ & $\begin{array}{c}\text { Extra-/ } \\
\text { intraabdominal } \\
\text { angiography }\end{array}$ & $\begin{array}{c}\text { Fluorescence } \\
\text { emission } \\
\text { source }\end{array}$ & Near-infrared system \\
\hline Aiba et al. [15] & 2020 & 110 & Colorectal resection & $0.1 \mathrm{mg} / \mathrm{kg}$ & 5 & Extraperitoneal & Xenon & OPAL1 (Karl Storz) \\
\hline Benčurik et al. [16] & 2020 & 100 & $\begin{array}{l}\text { Laparoscopic or robotic } \\
\text { LAR }\end{array}$ & $0.2 \mathrm{mg} / \mathrm{kg}$ & NA & Extraperitoneal & $\begin{array}{l}\text { Xenon } \\
\text { Laser }\end{array}$ & $\begin{array}{l}\text { SPIES (Karl Storz) } \\
\text { Firefly (Intuitive) }\end{array}$ \\
\hline Watanabe et al. [17] & 2020 & 236 & Laparoscopic LAR & $0.25 \mathrm{mg} / \mathrm{kg}$ & NA & Extraperitoneal & $\begin{array}{l}\text { Xenon } \\
\text { Laser }\end{array}$ & $\begin{array}{l}\text { D-light P (Karl Storz) } \\
1588 \text { AlM (Stryker) }\end{array}$ \\
\hline De Nardi et al. [18] & 2020 & 240 & $\begin{array}{l}\text { Laparoscopic left-sided } \\
\text { colorectal resection }\end{array}$ & $0.3 \mathrm{mg} / \mathrm{kg}$ & NA & NA & Xenon & IMAGE1 (Karl Storz) \\
\hline Son et al. [9] & 2019 & 86 & Laparoscopic AR or LAR & $0.25 \mathrm{mg} / \mathrm{kg}$ & NA & Extra- or intraperitoneal & Xenon & IMAGE1 S (Karl Storz) \\
\hline Ogino et al. [19] & 2019 & 74 & Colorectal surgery & Bolus $5 \mathrm{mg}$ & 15 & Extraperitoneal & LED & $\begin{array}{l}\text { Photodynamic eye system } \\
\text { (Hamamatsu Photonics) }\end{array}$ \\
\hline Morales-Conde et al. [20] & 2019 & 192 & Colorectal surgery & Bolus $15 \mathrm{mg}$ & 5 & Extraperitoneal & $\begin{array}{l}\text { Xenon } \\
\text { Laser }\end{array}$ & $\begin{array}{l}\text { IMAGE 1S (Karl Storz) } \\
1588 \text { AIM (Stryker) }\end{array}$ \\
\hline Chang et al. [21] & 2019 & 110 & NA & Bolus $5 \mathrm{mg}$ & NA & Extraperitoneal & Laser & SPY Elite System (Stryker) \\
\hline Boni et al. [22] & 2017 & 42 & Laparoscopic LAR & $0.2 \mathrm{mg} / \mathrm{kg}$ & NA & NA & Xenon & IMAGE1 (Karl Storz) \\
\hline
\end{tabular}

ICG, indocyanine green; LAR, low anterior resection; AR, anterior resection; LED, light-emitting diode; NA, not available.

Karl Storz, Tuttlingen, Germany; Intuitive, Sunnyvale, CA, USA; Stryker, Kalamazoo, MI, USA; Hamamatsu Photonics, Hamamatsu, Japan. 
In previous studies, ICG fluorescence has not been demonstrated to reduce anastomotic complications, but most studies have been based on the qualitative evaluation of ICG fluorescence in the colon wall or mucosa $[23,24]$. ICG angiography has not been verified as a predictor of anastomotic complications because qualitative evaluation alone is limited in accurately distinguishing changes in the microcirculation of the large intestine. Quantitative blood flow analysis is necessary to measure the changes in colonic microcirculation to predict bowel viability [25]. The quantitative analysis of colonic perfusion is still rare in surgical practice. In recent quantitative studies, $\mathrm{F}_{\text {MAX }}, \mathrm{T}_{1 / 2 M A X}$, and fluorescence slope were correlated with bowel viability or anastomotic leakage [2628]. In our study, factors related to perfusion time, such as $T_{M A X}$, $\mathrm{T}_{1 / 2 M A X}$, and perfusion time ratio were analyzed as significant predictors of anastomotic complications [9].

Interestingly, conditional factors, including distance, surrounding lighting, and fluorescence emission source, significantly affect the quantitative parameters, especially $\mathrm{F}_{\text {MAX }}, \mathrm{T}_{1 / 2 \mathrm{MAX}}$, and Slope in our in vitro study. The standardization protocol of ICG angiogra- phy can improve the quality and consistency of quantitative analysis, and an optimized protocol with an optimal distance of 4 to 5 $\mathrm{cm}$ using ICG specific modes can be recommended [11].

When our laparoscopic ICG protocol was applied to robot surgery, we could obtain similar fluorescence images using the Firefly in the Da Vinci XI (Intuitive, Sunnyvale, CA, USA), which is installed in our institution. From this personal experience for robotic surgery, laparoscopic ICG fluorescence protocol could be applied directly to robot surgery, because the Firefly in the Da Vinci XI is the equipment provided by Novadaq (Stryker), which has essentially the same laser NIR camera system.

\section{FLUORESCENCE LYMPH NODE NAVIGATION}

\section{Fluorescence lymph node mapping}

FLNM is a promising surgical application of image-guided surgery. When ICG is injected into the colonic wall, it spreads through the lymphatic drainage from the distal perivascular space with

Table 2. The protocols of fluorescence lymph node mapping

\begin{tabular}{|c|c|c|c|c|c|c|c|c|c|c|c|}
\hline Reference & Year & $\begin{array}{c}\text { No. of } \\
\text { patients }\end{array}$ & Dye & $\begin{array}{l}\text { Submucosal/ } \\
\text { subserosal }\end{array}$ & $\begin{array}{c}\text { ICG } \\
\text { dosage } \\
(\mathrm{mg})\end{array}$ & Waiting time & $\begin{array}{l}\text { Lymph } \\
\text { node } \\
\text { (mean) }\end{array}$ & $\begin{array}{l}\text { SLN } \\
\text { (mean) }\end{array}$ & $\begin{array}{c}\text { SLN } \\
\text { assessment }\end{array}$ & $\begin{array}{c}\text { Fluorescence } \\
\text { emission } \\
\text { source }\end{array}$ & Near-infrared system \\
\hline Son et al. [10] & 2021 & 192 & ICG & Submucosal & 1 & $16-18 \mathrm{hr}$ & 33.3 & NA & In vivo & Laser/xenon & $\begin{array}{l}1588 \text { AIM (Stryker)/ } \\
\text { IMAGE1 (Karl storz) }\end{array}$ \\
\hline Park et al. [31] & 2020 & 25 & ICG & Submucosal & $2.5-10$ & $3-24 \mathrm{hr}$ & 41.0 & NA & In vivo & Xenon & D-light P (Karl storz) \\
\hline $\begin{array}{l}\text { Ankersmit et al. } \\
\text { [34] }\end{array}$ & 2019 & 30 & ICG & $\begin{array}{r}\text { Submucosal/ } \\
\text { subserosal }\end{array}$ & 20 & 3-10 min & 13.0 & 3.5 & In vivo & Xenon & NIR-imaging system (Olympus) \\
\hline Currie et al. [35] & 2017 & 30 & ICG & Submucosal & 2.5 & $7 \mathrm{~min}$ & 34.0 & 3.0 & In vivo & Xenon & NIR-imaging system (Olympus) \\
\hline $\begin{array}{l}\text { Andersen et al. } \\
\text { [36] }\end{array}$ & 2017 & 29 & ICG & Subserosal & 5 & $20 \mathrm{~min}$ & 24.0 & 1.0 & $\begin{array}{l}\text { In vivo/ } \\
\text { ex vivo }\end{array}$ & Xenon & SPIES (Karl storz) \\
\hline Weixler et al. [37] & 2017 & 50 & $\operatorname{IRD}$ & Subserosal & 6 & NA & 17.5 & 10.4 & Ex vivo & Laser & Mini-FLARE \\
\hline $\begin{array}{l}\text { Watanabe et al. } \\
\text { [38] }\end{array}$ & 2016 & 20 & ICG & Subserosal & 2 & $30 \mathrm{~min}$ & 17.5 & 10.4 & In vivo & Xenon & D-light P (Karl storz) \\
\hline Liberale et al. [39] & 2016 & 12 & ICG & Subserosal & 2 & $152 \mathrm{~min}$ & 22.4 & 1.5 & Ex vivo & LED & $\begin{array}{c}\text { Photodynamic eye system } \\
\text { (Hamamatsu Photonics) }\end{array}$ \\
\hline $\begin{array}{l}\text { Schaafsma et al. } \\
\text { [40] }\end{array}$ & 2013 & 22 & $\operatorname{IRD}$ & Submucosal & 4 & NA & 20.5 & 3.5 & Ex vivo & Laser & Mini-FLARE \\
\hline Hirche et al. [33] & 2012 & 26 & ICG & Subserosal & $5-20$ & $3-10 \mathrm{~min}$ & 32.9 & 1.7 & In vivo & Xenon & $\begin{array}{l}\text { IC-view (Pulsion Medical } \\
\text { System) }\end{array}$ \\
\hline Cahill et al. [7] & 2012 & 18 & ICG & Submucosal & $5-7.5$ & NA & NA & 3.6 & In vivo & Xenon & CE approved device (Olympus) \\
\hline $\begin{array}{l}\text { Hutteman et al. } \\
\text { [41] }\end{array}$ & 2011 & 19 & $\mathbb{R D}$ & Submucosal & 1 & NA & 16.2 & 3.2 & Ex vivo & Laser & Mini-FLARE \\
\hline Noura et al. [32] & 2010 & 25 & ICG & Submucosal & 5 & $30 \mathrm{~min}$ & NA & 2.1 & In vivo & LED & $\begin{array}{c}\text { Photodynamic eye system } \\
\text { (Hamamatsu Photonics) }\end{array}$ \\
\hline Kusano et al. [8] & 2008 & 26 & ICG & Subserosal & NA & NA & NA & 2.4 & In vivo & LED & $\begin{array}{l}\text { Photodynamic eye system } \\
\text { (Hamamatsu Photonics) }\end{array}$ \\
\hline
\end{tabular}

ICG, indocyanine green; IRD, IRDye800CW; SLN, sentinel lymph node; CE, Conformite Europeenne; NA, not available.

Karl Storz, Tuttlingen, Germany; Olympus, Tokyo, Japan; Pulsion Medical System, Feldkirchen, Germany; Hamamatsu Photonics, Hamamatsu, Japan. 
slow interstitial fluid reabsorption [29]. In colorectal cancer surgery, FLNM helps the surgeon visualize the lymphatic pathway to perform a more accurate dissection of the lymph nodes [30]. The patients' body mass index (BMI) can affect the success of FLNM because higher BMI means more visceral fat tissue and that can disturb the fluorescence signals of lymph nodes that are covered with thick mesenteric fat tissue [10]. FLNM protocols have been introduced from previous studies and summarized in Table $2[7$, $8,10,31-41]$.

In the comparison study between the ICG FLNM group and the conventional group, the total number of harvested lymph nodes was significantly higher with FLNM [31]. Being able to visualize the lymph nodes and lymphatic drainage pathway, the surgeon can dissect lymph nodes much more radically. When a fluorescent lymph node is found in the extraterritorial area outside of the conventional radical dissection plane, a surgeon can extend the dissecting plane for complete fluorescence lymph node removal. From the oncological point of view, at least 12 lymph nodes should be retrieved to evaluate the pathologic stage of cancer, so that patients may receive proper chemotherapy after surgery. FLNM can be considered as one of the surgical tools for the retrieval of an adequate number of lymph nodes.

Although the total number of harvested lymph nodes was greater in the case of successful FLNM, there is no effect on the total number of metastatic lymph nodes in our study. The previous study on comparison of clinical outcomes between ICGguided laparoscopic right hemicolectomy and conventional surgery have found that harvested lymph nodes were greater but no difference in metastatic lymph nodes by the ICG-guided surgery [42]. In other words, lymph node harvest is a function of pathology. Another possible explanation is that the pathologist took greater effort on ICG-labeled lymph nodes that the surgeon had isolated during surgery. The other possible explanation is that lymph nodes that might have been omitted by the conventional way were harvested with the help of visual aids. Thus, it can be suggested that FLNM helps harvest more lymph nodes than the conventional method. Therefore, the successful FLNM could be expected not only to help isolate more lymph nodes but also to effective pathologic isolation of D3 lymph nodes after radical lymphadenectomy [10].

However, FLNM using ICG has a fundamental limitation that has not yet been solved. FLNM would be a poor modality to identify metastatic lymph nodes. ICG binds to macrophages within the lymph nodes, but normal lymphoid tissues are replaced by the cancer cells in the metastatic lymph node. So, the metastatic lymph nodes would drain ICG less easily, that none or crescentshaped fluorescence would appear under the NIR camera. In a recent study in Japan, they explored the metastatic lymph nodes under a microscopic NIR camera. Fluorescence did not emit light in the area occupied by cancer cells, and crescent-shaped fluorescence was observed in the node where some lymphoid tissue still has remained [43]. In advanced colorectal cancer, FLNM still has limitations in accuracy and reliability on creating cancer cell spreading pathways. The detection of the metastatic lymph nodes is still challenging for ICG FLNM. However, in early staged cancer, since ICG submucosal injection can be performed closer to the lesion, it is expected that FLNM will be possible as sentinel lymph node (SLN) mapping.

\section{Sentinel lymph node mapping}

The original concept of the SLN is the hypothetical lymph nodes to which primary cancer initially drains. In many fields of cancer, especially in breast cancer and malignant melanoma, ICG is used as one of the tools for detecting SLNs to minimize surgical procedures and prevent postoperative complications [44]. The SLN mapping has promising results in cervical cancer, endometrial cancer, and gastrointestinal cancer [32, 45]. So far, SLN mapping has been attempted using blue dye or radioisotope. However, the success rate of lymph node mapping did not meet expectations using blue dye. With radioisotope usage, radiation exposure and additional assistance from nuclear physicians were required. With the development of the surgical NIR camera system, the ICG lymph node mapping has been tried for the detection of SLNs $[33,46]$.

SLN mapping with ICG can be inaccurate because the lymph nodes are replaced with tumors and thus lymphatic flow drains less in the lymph node-positive patients. From the ICG FLNM, the pathway of lymph nodes that connected with the primary tumor, however, within metastatic lymph nodes, the lymphatic flow could be blocked by infiltrated cancer cells. This phenomenon is visually shown by metastatic lymph nodes with insufficient uptake of ICG, which forms none or crescent-shaped fluorescence. In early cancer, submucosal ICG injection can be performed close to the tumor without disturbance of lymphatic flow, ICG stained lymph node mapping could be interpreted as SLN mapping.

For in vivo SLN mapping, ICG is usually injected into a patient after anesthetic induction in the operating room. After 10 to 30 minutes of ICG injection, fluorescence expressing lymph nodes are identified and marked using clip or suture tie for later pathological sentinel node examination. Some researchers used ex vivo SLN mapping through subserosal injection of fluorescence dye. In the cases of ex vivo SLN mapping, other non-FDA-approved fluorescence agents, such as IRDye800CW (Li-Cor, Lincoln, NE, USA) can be injected into colon tissue. The colonic layer should be selected as a subserosal or submucosal layer for ICG injection. The subserosal ICG injection can be directly performed by a surgeon using a butterfly needle, but when the needle tip enters into the muscular propria, ICG solution could not be shooted and ICG spillage should cause ICG contamination in the abdominal cavity. The submucosal layer can be used through a colonoscopy, reducing the possibility of ICG spillage. It is important to consider the spread of ICG solution in the submucosal layer to most accurately reproduce the lymphatic flow of early colon cancer with submucosal invasion. On the meta-analysis, colon cancer was analyzed to be more suitable for SLN detection than rectal cancer 
[46]. Interestingly, a recent study reported that the staining lymph nodes of ICG and blue dye injected into the submucosal layer and subserosal layer, respectively, did not match. Since the lymphatic pathways of the submucosal layer and the subserosal layer are different, the submucosal injection would be appropriate to detect SLN in early colon cancer. The most important limitation of SLNM is the false-negative rates of more than $20 \%$ of lymph node metastasis. In colon cancer, the false-negative value of SLN mapping was associated with less than 3 SLNs and tumor-replaced lymph nodes. In addition, SLN mapping was successfully achieved in colon cancer than rectal cancer [47]. This association between the number of SLNs harvested and false-negative rates may be related to the different anatomic patterns of lymphatic drainage of the colon, the skin (melanoma), and the breast. While the lymphatic drainage is in conduit or funnel-shaped pattern passing through 1 or 2 sentinel nodes as first echelon lymph nodes in the skin or breast tissue, and metastatic cancer cells can be spreading into second and third echelon lymph nodes. But, the lymphatic drainage of the colon segment might be quite the opposite anatomical structure. A broad distributed base of lymphatics can be drained into a group of sentinel nodes as the first echelon lymph node, and then converging into 1 or 2 apical lymph nodes at the base of the mesenteric artery, which is considered as the typical drainage pattern of colonic lymphatics. Therefore, securing 3 or more SLNs in the first echelon lymph nodes is likely to be a prerequisite for SLN mapping to successfully proceed in future clinical studies for patients with early colon cancer [47].

Besides, the range of the lymph node dissection with FLNM is still undetermined in colorectal cancer surgery. The SLN mapping is still difficult to define in colorectal cancer, because of the dual lymphatic flow and existence of skipped lymph nodes.

There are still a lot of questions to be solved, but we believe that ICG FLNM could be one of the important methods for finding SLNs in the selected patients. Therefore, future ICG lymph node mapping studies will serve as the basis for establishing SLN mapping in early colon cancer.

\section{CONCLUSION}

The multifunctional ICG applications for fluorescent tumor localization, successful FLNM, and ICG angiography can be performed within a single surgery under a laparoscopic or robotic NIR-imaging system. The standardization of multifunctional ICG applications should be established for fluorescence-guided laparoscopic colorectal surgery.

\section{CONFLICT OF INTEREST}

No potential conflict of interest relevant to this article was reported.

\section{ACKNOWLEDGMENTS}

This study was supported by a 2020 research grant from Pusan National University Yangsan Hospital.

\section{REFERENCES}

1. Van Manen L, Handgraaf HJ, Diana M, Dijkstra J, Ishizawa T, Vahrmeijer AL, et al. A practical guide for the use of indocyanine green and methylene blue in fluorescence-guided abdominal surgery. Journal of surgical oncology 2018;118:283-300.

2. Park JH, Moon HS, Kwon IS, Yun GY, Lee SH, Park DH, et al. Usefulness of colonic tattooing using indocyanine green in patients with colorectal tumors. World J Clin Cases 2018;6:632-40.

3. Reinhart MB, Huntington CR, Blair LJ, Heniford BT, Augenstein VA. Indocyanine green: historical context, current applications, and future considerations. Surg Innov 2016;23:166-75

4. Liberale G, Bourgeois P, Larsimont D, Moreau M, Donckier V, Ishizawa $\mathrm{T}$. Indocyanine green fluorescence-guided surgery after IV injection in metastatic colorectal cancer: a systematic review. Eur J Surg Oncol 2017;43:1656-67.

5. Watanabe M, Tsunoda A, Narita K, Kusano M, Miwa M. Colonic tattooing using fluorescence imaging with light-emitting diodeactivated indocyanine green: a feasibility study. Surg Today 2009; 39:214-8.

6. Trakarnsanga A, Akaraviputh T. Endoscopic tattooing of colorectal lesions: is it a risk-free procedure? World J Gastrointest Endosc 2011;3:256-60.

7. Cahill RA, Anderson M, Wang LM, Lindsey I, Cunningham C, Mortensen NJ. Near-infrared (NIR) laparoscopy for intraoperative lymphatic road-mapping and sentinel node identification during definitive surgical resection of early-stage colorectal neoplasia. Surg Endosc 2012;26:197-204.

8. Kusano M, Tajima Y, Yamazaki K, Kato M, Watanabe M, Miwa M. Sentinel node mapping guided by indocyanine green fluorescence imaging: a new method for sentinel node navigation surgery in gastrointestinal cancer. Dig Surg 2008;25:103-8

9. Son GM, Kwon MS, Kim Y, Kim J, Kim SH, Lee JW. Quantitative analysis of colon perfusion pattern using indocyanine green (ICG) angiography in laparoscopic colorectal surgery. Surg Endosc 2019; 33:1640-9.

10. Ahn HM, Son GM, Lee IY, Shin DH, Kim TK, Park SB, et al. Optimal ICG dosage of preoperative colonoscopic tattooing for fluorescence-guided laparoscopic colorectal surgery. Surg Endosc 2021 Feb 26 [Epub]. https://doi.org/10.1007/s00464-021-08382-5.

11. Ahn HM, Son GM, Lee IY, Park SH, Kim NS, Baek KR. Optimization of Indocyanine Green (ICG) angiography for colon perfusion during laparoscopic colorectal surgery. Colorectal Dis 2021 Apr 24 [Epub]. https://doi.org/10.1111/codi.15684.

12. Price N, Gottfried MR, Clary E, Lawson DC, Baillie J, Mergener K, et al. Safety and efficacy of India ink and indocyanine green as colonic tattooing agents. Gastrointest Endosc 2000;51(4 Pt 1):438- 
42.

13. Elias E, Mukherji D, Faraj W, Khalife M, Dimassi H, Eloubeidi M, et al. Lymph-node ratio is an independent prognostic factor in patients with stage III colorectal cancer: a retrospective study from the Middle East. World J Surg Oncol 2012;10:63.

14. Nishigori N, Koyama F, Nakagawa T, Nakamura S, Ueda T, Inoue $\mathrm{T}$, et al. Visualization of lymph/blood flow in laparoscopic colorectal cancer surgery by ICG fluorescence imaging (Lap-IGFI). Ann Surg Oncol 2016;23 Suppl 2:S266-74.

15. Aiba T, Uehara K, Ogura A, Tanaka A, Yonekawa Y, Hattori N, et al. The significance of the time to arterial perfusion in intraoperative ICG angiography during colorectal surgery. Surg Endosc 2021 Jan 4 [Epub]. https://doi.org/10.1007/s00464-020-08185-0.

16. Benčurik V, Škrovina M, Martínek L, Bartoš J, Macháčková M, Dosoudil M, et al. Intraoperative fluorescence angiography and risk factors of anastomotic leakage in mini-invasive low rectal resections. Surg Endosc 2020 Sep 24 [Epub]. https://doi.org/10.1007/ s00464-020-07982-X.

17. Watanabe J, Ishibe A, Suwa Y, Suwa H, Ota M, Kunisaki C, et al. Indocyanine green fluorescence imaging to reduce the risk of anastomotic leakage in laparoscopic low anterior resection for rectal cancer: a propensity score-matched cohort study. Surg Endosc 2020;34:202-8.

18. De Nardi P, Elmore U, Maggi G, Maggiore R, Boni L, Cassinotti E, et al. Intraoperative angiography with indocyanine green to assess anastomosis perfusion in patients undergoing laparoscopic colorectal resection: results of a multicenter randomized controlled trial. Surg Endosc 2020;34:53-60.

19. Ogino T, Hata T, Kawada J, Okano M, Kim Y, Okuyama M, et al. The risk factor of anastomotic hypoperfusion in colorectal surgery. J Surg Res 2019;244:265-71.

20. Morales-Conde S, Alarcón I, Yang T, Licardie E, Camacho V, Aguilar Del Castillo F, et al. Fluorescence angiography with indocyanine green (ICG) to evaluate anastomosis in colorectal surgery: where does it have more value? Surg Endosc 2020;34:3897907.

21. Chang YK, Foo CC, Yip J, Wei R, Ng KK, Lo O, et al. The impact of indocyanine-green fluorescence angiogram on colorectal resection. Surgeon 2019;17:270-6.

22. Boni L, Fingerhut A, Marzorati A, Rausei S, Dionigi G, Cassinotti E. Indocyanine green fluorescence angiography during laparoscopic low anterior resection: results of a case-matched study. Surg Endosc 2017;31:1836-40.

23. Nachiappan S, Askari A, Currie A, Kennedy RH, Faiz O. Intraoperative assessment of colorectal anastomotic integrity: a systematic review. Surg Endosc 2014;28:2513-30.

24. Sherwinter DA, Gallagher J, Donkar T. Intra-operative transanal near infrared imaging of colorectal anastomotic perfusion: a feasibility study. Colorectal Dis 2013;15:91-6.

25. Gröne J, Koch D, Kreis ME. Impact of intraoperative microperfusion assessment with Pinpoint Perfusion Imaging on surgical management of laparoscopic low rectal and anorectal anastomo- ses. Colorectal Dis 2015;17 Suppl 3:22-8.

26. Kamiya K, Unno N, Miyazaki S, Sano M, Kikuchi H, Hiramatsu Y, et al. Quantitative assessment of the free jejunal graft perfusion. J Surg Res 2015;194:394-9.

27. Protyniak B, Dinallo AM, Boyan WP Jr, Dressner RM, Arvanitis ML. Intraoperative indocyanine green fluorescence angiography: an objective evaluation of anastomotic perfusion in colorectal surgery. Am Surg 2015;81:580-4.

28. Wada T, Kawada K, Takahashi R, Yoshitomi M, Hida K, Hasegawa $S$, et al. ICG fluorescence imaging for quantitative evaluation of colonic perfusion in laparoscopic colorectal surgery. Surg Endosc 2017;31:4184-93.

29. Ietto G, Amico F, Soldini G, Chiappa C, Franchin M, Iovino D, et al. Real-time intraoperative fluorescent lymphography: a new technique for lymphatic sparing surgery. Transplant Proc 2016;48: 3073-8.

30. Joshi HM, Keller DS, Chand M. Utilization of Indocyanine green to demonstrate lymphatic mapping in colon cancer. J Surg Oncol 2017;116:1005-7.

31. Park SY, Park JS, Kim HJ, Woo IT, Park IK, Choi GS. Indocyanine green fluorescence imaging-guided laparoscopic surgery could achieve radical D3 dissection in patients with advanced right-sided colon cancer. Dis Colon Rectum 2020;63:441-9.

32. Noura S, Ohue M, Seki Y, Tanaka K, Motoori M, Kishi K, et al. Feasibility of a lateral region sentinel node biopsy of lower rectal cancer guided by indocyanine green using a near-infrared camera system. Ann Surg Oncol 2010;17:144-51.

33. Hirche C, Mohr Z, Kneif S, Doniga S, Murawa D, Strik M, et al. Ultrastaging of colon cancer by sentinel node biopsy using fluorescence navigation with indocyanine green. Int J Colorectal Dis 2012;27:319-24.

34. Ankersmit M, Bonjer HJ, Hannink G, Schoonmade LJ, van der Pas MH, Meijerink WJ. Near-infrared fluorescence imaging for sentinel lymph node identification in colon cancer: a prospective single-center study and systematic review with meta-analysis. Tech Coloproctol 2019;23:1113-26.

35. Currie AC, Brigic A, Thomas-Gibson S, Suzuki N, Moorghen M, Jenkins JT, et al. A pilot study to assess near infrared laparoscopy with indocyanine green (ICG) for intraoperative sentinel lymph node mapping in early colon cancer. Eur J Surg Oncol 2017;43: 2044-51.

36. Andersen HS, Bennedsen AL, Burgdorf SK, Eriksen JR, Eiholm S, Toxvaerd A, et al. In vivo and ex vivo sentinel node mapping does not identify the same lymph nodes in colon cancer. Int J Colorectal Dis 2017;32:983-90.

37. Weixler B, Rickenbacher A, Raptis DA, Viehl CT, Guller U, Rueff J, et al. Sentinel lymph node mapping with isosulfan blue or indocyanine green in colon cancer shows comparable results and identifies patients with decreased survival: a prospective singlecenter trial. World J Surg 2017;41:2378-86.

38. Watanabe J, Ota M, Suwa Y, Ishibe A, Masui H, Nagahori K. Realtime indocyanine green fluorescence imaging-guided complete 
mesocolic excision in laparoscopic flexural colon cancer surgery. Dis Colon Rectum 2016;59:701-5.

39. Liberale G, Vankerckhove S, Galdon MG, Larsimont D, Ahmed B, Bouazza F, et al. Sentinel lymph node detection by blue dye versus indocyanine green fluorescence imaging in colon cancer. Anticancer Res 2016;36:4853-8.

40. Schaafsma BE, Verbeek FP, van der Vorst JR, Hutteman M, Kuppen PJ, Frangioni JV, et al. Ex vivo sentinel node mapping in colon cancer combining blue dye staining and fluorescence imaging. J Surg Res 2013;183:253-7.

41. Hutteman M, Choi HS, Mieog JS, van der Vorst JR, Ashitate Y, Kuppen PJ, et al. Clinical translation of ex vivo sentinel lymph node mapping for colorectal cancer using invisible near-infrared fluorescence light. Ann Surg Oncol 2011;18:1006-14.

42. Vogel JD, Eskicioglu C, Weiser MR, Feingold DL, Steele SR. The American Society of Colon and Rectal Surgeons Clinical Practice Guidelines for the Treatment of Colon Cancer. Dis Colon Rectum 2017;60:999-1017.

43. Kakizoe M, Watanabe J, Suwa Y, Nakagawa K, Suwa H, Ozawa M, et al. The histopathological evaluation based on the indocyanine green fluorescence imaging of regional lymph node metastasis of splenic flexural colon cancer by near-infrared observation. Int J Colorectal Dis 2021;36:717-23.

44. Liberale G, Bohlok A, Bormans A, Bouazza F, Galdon MG, El Nakadi I, et al. Indocyanine green fluorescence imaging for sentinel lymph node detection in colorectal cancer: a systematic review. Eur J Surg Oncol 2018;44:1301-6.

45. Bedyńska M, Szewczyk G, Klepacka T, Sachadel K, Maciejewski T, Szukiewicz D, et al. Sentinel lymph node mapping using indocyanine green in patients with uterine and cervical neoplasms: restrictions of the method. Arch Gynecol Obstet 2019;299:1373-84.

46. Villegas-Tovar E, Jimenez-Lillo J, Jimenez-Valerio V, Diaz-GironGidi A, Faes-Petersen R, Otero-Piñeiro A, et al. Performance of Indocyanine green for sentinel lymph node mapping and lymph node metastasis in colorectal cancer: a diagnostic test accuracy meta-analysis. Surg Endosc 2020;34:1035-47.

47. Nissan A, Protic M, Bilchik A, Eberhardt J, Peoples GE, Stojadinovic A. Predictive model of outcome of targeted nodal assessment in colorectal cancer. Ann Surg 2010;251:265-74. 